\title{
Two Variable Cubic Spline Interpolation
}

\author{
Dhanya Ramachandran ${ }^{1}$, Dr.V. Madhukar Mallayya ${ }^{2}$ \\ ${ }^{I}$ Assistant Professor (Maths) Mar Baselios College ofEngg. \& Tech. Nalanchira, Trivandrum \\ ${ }^{2}$ Professor \& Head (Maths) Mohandas College of Engg. \& Tech. Anad, Trivandrum
}

\begin{abstract}
Two variable natural cubic spline interpolation formula is derived and illustrated using an example Keywords: Two variable interpolation, Two variable spline, Natural spline Subject Classification (2000) 65007
\end{abstract}

\section{Introduction}

If the explicit nature of the function $y=f(x)$ is not known but the set of tabular values satisfying the function is known then the process of replacing $f(x)$ by suitable function say $\mathrm{g}(\mathrm{x})$ satisfying the given set of tabular values is called interpolation. If $\mathrm{g}(\mathrm{x})$ is a polynomial then it is called polynomial interpolation [4]. In many cases it is seen that polynomial oscillates varyingly but the function varies smoothly [1]. To overcome this spline function is considered which is a function of polynomial bits joined together. The cubic spline procedure has sufficient flexibility due to the four constantsinvolved in a general cubic polynomial which ensures the condition that the interpolant is continuously differentiable in the interval and has continuous second derivative [3]. Thus because of their smoothness conditions the most frequently used spline interpolation is the cubic spline interpolation. A two variable cubic spline interpolation of a function $z=f(x, y)$ is the fitting of a unique series of cubic splines for a given set of data points $\left(x_{i}, y_{j}, z_{i j}\right)$. The points $(\mathrm{x}, \mathrm{y})$ at which $\mathrm{f}(\mathrm{x}, \mathrm{y})$ are known lie on a grid in the $x-y$ plane. In order to derive a two variable natural cubic spline the existence of continuity condition of the spline function and its partial derivatives at the edge of each grid are assumed.

\section{Two Variable Natural Cubic Spline}

Consider the division rectangle $I=[a, b] \times[c, d]$. Let $a=x_{1}<x_{2}<----<x_{n}=b$ and $c=y_{1}<y_{2}<----<y_{m}=d$ be the set of data points satisfied by $f\left(x_{i}, y_{j}\right)=z_{i j}$ for all $\mathrm{i}=1,2, \ldots, \mathrm{n}$ and $\mathrm{j}=1,2, \ldots . ., \mathrm{m}$.[2] . A two variable cubic spline $S_{i j}(x, y)$ is a unique function coinciding with $z_{i j}$ in each rectangular grid $I_{i j}=\left[x_{i}, x_{i+1}\right] \times\left\lfloor y_{j}, y_{j+1}\right\rfloor$ for all $\mathrm{i}=1,2, \ldots,(\mathrm{n}-1)$ and $\mathrm{j}=1,2, \ldots \ldots,(\mathrm{m}-1)$. Since $S_{i j}(x, y)$ is a cubic spline in two variables its all second order partial derivatives should be linear and continuous. Here we are considering the second order partial derivative with respect to $\mathrm{x}$

Let $\frac{\partial^{2} S_{i j}}{\partial x^{2}}=\frac{M_{i}\left(x_{i+1}-x\right)}{h_{i}}+\frac{M_{i+1}\left(x-x_{i}\right)}{h_{i}}+\frac{N_{j}\left(y_{j+1}-y\right)}{k_{j}}+\frac{N_{j+1}\left(y-y_{j}\right)}{k_{j}}$

$\left.\begin{array}{l}\text { Let } x_{i+1}-x_{i}=h_{i} \\ y_{j+1}-y_{j}=k_{j}\end{array}\right\} \begin{aligned} & \mathrm{i}=1,2,---, \mathrm{n}-1 \\ & \mathrm{j}=1,2,----, \mathrm{m}-1\end{aligned}$

Integrating (1) with respect to $\mathrm{x}$,

$\frac{\partial S_{i j}}{\partial x}=-\frac{M_{i}\left(x_{i+1}-x\right)^{2}}{2 h_{i}}+\frac{M_{i+1}\left(x-x_{i}\right)^{2}}{2 h_{i}}+\frac{N_{j}\left(y_{j+1}-y\right)\left(x-x_{i}\right)}{k_{j}}+\frac{N_{j+1}\left(y-y_{j}\right)\left(x_{i+1}-x\right)}{k_{j}}+A\left(y_{j+1}-y\right)+B$

Integrating (2) with respect to $\mathrm{x}$,

$$
\begin{aligned}
S_{i j}= & \frac{M_{i}\left(x_{i+1}-x\right)^{3}}{6 h_{i}}+\frac{M_{i+1}\left(x-x_{i}\right)^{3}}{6 h_{i}}+\frac{N_{j}\left(y_{j+1}-y\right)\left(x-x_{i}\right)^{2}}{2 k_{j}}-\frac{N_{j+1}\left(y-y_{j}\right)\left(x_{i+1}-x\right)^{2}}{2 k_{j}}+ \\
& A\left(y_{j+1}-y\right)\left(x-x_{i}\right)+B\left(x_{i+1}-x\right)+C\left(y-y_{j}\right)+D
\end{aligned}
$$


Since the spline interpolates at the knots:
(i) $S_{i j}\left(x_{i+1}, y_{j}\right)=z_{i+1, j}$
(ii) $S_{i j}\left(x_{i}, y_{j+1}\right)=z_{i, j+1}$
(iii) $S_{i j}\left(x_{i}, y_{j}\right)=z_{i, j}$
(iv) $S_{i j}\left(x_{i+1}, y_{j+1}\right)=z_{i+1, j+1}$

Applying the conditions (i), (ii), (iii), (iv) to (3)

$$
\begin{aligned}
& z_{i+1, j}=\frac{M_{i+1} h_{i}^{2}}{6}+\frac{N_{j} h_{i}^{2}}{2}+A h_{i} k_{j}+D \\
& z_{i, j+1}=\frac{M_{i} h_{i}^{2}}{6}-\frac{N_{j+1} h_{i}^{2}}{2}+B h_{i}+C k_{j}+D \\
& z_{i, j}=\frac{M_{i} h_{i}^{2}}{6}+B h_{i}+D
\end{aligned}
$$

$z_{i+1, j+1}=\frac{M_{i+1} h_{i}^{2}}{6}+C k_{j}+D$

Solving (4), (5), (6), (7) we get the following constants

$$
\begin{aligned}
& A=\frac{\left(z_{i+1, j}-z_{i j}\right)}{h_{i} k_{j}}-\frac{\left(z_{i+1, j+1}-z_{i, j+1}\right)}{h_{i} k_{j}}+\frac{\left(N_{j+1}-N_{j}\right) h_{i}}{2 k_{j}} \\
& B=\frac{\left(M_{i+1}-M_{i}\right) h_{i}}{6}+\frac{N_{j+1} h_{i}}{2}-\frac{\left(z_{i+1, j+1}-z_{i, j+1}\right)}{h_{i}} \\
& C=\frac{\left(z_{i, j+1}-z_{i j}\right)}{k_{j}}+\frac{N_{j+1} h_{i}^{2}}{2 k_{j}} \\
& D=\left(z_{i+1, j+1}-z_{i, j+1}\right)+z_{i j}-\left(\frac{M_{i+1}}{6}+\frac{N_{j+1}}{2}\right) h_{i}{ }^{2}
\end{aligned}
$$

So from (3) the two variable cubic spline is

$$
\begin{aligned}
S_{i j}= & \frac{M_{i}\left(x_{i+1}-x\right)^{3}}{6 h_{i}}+\frac{M_{i+1}\left(x-x_{i}\right)^{3}}{6 h_{i}}+\frac{N_{j}\left(y_{j+1}-y\right)\left(x-x_{i}\right)^{2}}{2 k_{j}}-\frac{N_{j+1}\left(y-y_{j}\right)\left(x_{i+1}-x\right)^{2}}{2 k_{j}} \\
& +\left\{\frac{\left(z_{i+1, j}-z_{i j}\right)}{h_{i} k_{j}}-\frac{\left(z_{i+1, j+1}-z_{i, j+1}\right)}{h_{i} k_{j}}+\frac{\left(N_{j+1}-N_{j}\right) h_{i}}{2 k_{j}}\right\}\left(y_{j+1}-y\right)\left(x-x_{i}\right)+ \\
& \left\{\frac{\left(M_{i+1}-M_{i}\right) h_{i}}{6}+\frac{N_{j+1} h_{i}}{2}-\frac{\left(z_{i+1, j+1}-z_{i, j+1}\right)}{h_{i}}\right\}\left(x_{i+1}-x\right)+ \\
& \left\{\frac{\left(z_{i, j+1}-z_{i j}\right)}{k_{j}}+\frac{N_{j+1} h_{i}^{2}}{2 k_{j}}\right\}\left(y-y_{j}\right)+\left\{\left(z_{i+1, j+1}-z_{i, j+1}\right)+z_{i j}-\left(\frac{M_{i+1}}{6}+\frac{N_{j+1}}{2}\right) h_{i}^{2}\right\},
\end{aligned}
$$

$\forall(x, y) \in\left[x_{i}, x_{i+1}\right] \times\left[y_{j}, y_{j+1}\right], \forall i=1,2, \ldots . .,(n-1), \forall j=1,2, \ldots \ldots .(m-1)$

In two variable spline function there exist a unique tangent plane at the two surfaces in every node. So

corresponding to the node $\left(x_{i+1}, y_{j}\right)$ we have $\frac{\partial S_{i j}}{\partial x}\left(x_{i+1}, y_{j}\right)=\frac{\partial S_{i+1, j}}{\partial x}\left(x_{i+1}, y_{j}\right)$

$\frac{\partial S_{i j}}{\partial x}(x, y)=-\frac{M_{i}\left(x_{i+1}-x\right)^{2}}{2 h_{i}}+\frac{M_{i+1}\left(x-x_{i}\right)^{2}}{2 h_{i}}+\frac{N_{j}\left(y_{j+1}-y\right)\left(x-x_{i}\right)}{k_{j}}+\frac{N_{j+1}\left(y-y_{j}\right)\left(x_{i+1}-x\right)}{k_{j}}$ 


$$
\begin{aligned}
+\left\{\frac{\left(z_{i+1, j}-z_{i j}\right)}{h_{i} k_{j}}-\frac{\left(z_{i+1, j+1}-z_{i, j+1}\right)}{h_{i} k_{j}}+\frac{\left(N_{j+1}-N_{j}\right) h_{i}}{2 k_{j}}\right\}\left(y_{j+1}-y\right) & \left.-\frac{\left(M_{i+1}-M_{i}\right) h_{i}}{6}+\frac{N_{j+1} h_{i}}{2}-\frac{\left(z_{i+1, j+1}-z_{i, j+1}\right)}{h_{i}}\right\} \\
& \left\{\frac{M_{i}}{6},\right.
\end{aligned}
$$

$\frac{\partial S_{i j}}{\partial x}\left(x_{i+1}, y_{j}\right)=\frac{M_{i+1} h_{i}}{3}+\frac{N_{j} h_{i}}{2}+\frac{z_{i+1, j}-z_{i j}}{h_{i}}+\frac{M_{i} h_{i}}{6}$

$\frac{\partial S_{i+1, j}}{\partial x}(x, y)=-\frac{M_{i+1}\left(x_{i+2}-x\right)^{2}}{2 h_{i+1}}+\frac{M_{i+2}\left(x-x_{i+1}\right)^{2}}{2 h_{i+1}}+\frac{N_{j}\left(y_{j+1}-y\right)\left(x-x_{i+1}\right)}{k_{j}}+$

$\frac{N_{j+1}\left(y-y_{j}\right)\left(x_{i+1}-x\right)}{k_{j}}+\left\{\frac{\left(z_{i+1, j}-z_{i j}\right)}{h_{i} k_{j}}-\frac{\left(z_{i+1, j+1}-z_{i, j+1}\right)}{h_{i} k_{j}}+\frac{\left(N_{j+1}-N_{j}\right) h_{i}}{2 k_{j}}\right\}\left(y_{j+1}-y\right)$

$-\left\{\frac{\left(M_{i+1}-M_{i}\right) h_{i}}{6}+\frac{N_{j+1} h_{i}}{2}-\frac{\left(z_{i+1, j+1}-z_{i, j+1}\right)}{h_{i}}\right\}$

$\frac{\partial S_{i+1, j}}{\partial x}\left(x_{i+1}, y_{j}\right)=-\frac{M_{i+1}}{3} h_{i+1}+\frac{z_{i+2, j}}{h_{i+1}}-\frac{z_{i+1, j}}{h_{i+1}}-\frac{M_{i+2}}{6} h_{i+1}-\frac{N_{j}}{2} h_{i+1}$

Equating (9) and (10)

$\frac{\left(z_{i+2, j}-z_{i+1, j}\right)}{h_{i+1}}-\frac{\left(z_{i+1, j}-z_{i j}\right)}{h_{i}}=\frac{M_{i+1}}{3}\left(h_{i}+h_{i+1}\right)+\frac{N_{j}}{2}\left(h_{i}+h_{i+1}\right)+\frac{M_{i} h_{i}}{6}+\frac{M_{i+2}}{6} h_{i+1}$

Assume $\frac{z_{i+1, j}-z_{i j}}{h_{i}}=\sigma_{i, j} \quad$ where $\mathrm{i}=1,2,----,(\mathrm{n}-1)$ and $\mathrm{j}=1,2,-----,(\mathrm{m}-1)$

$\frac{z_{i+2, j}-z_{i+1, j}}{h_{i+1}}=\sigma_{i+1, j}$

Assume $\lambda_{i}=\frac{h_{i+1}}{h_{i}+h_{i+1}}$ where $\mathrm{i}=1,2,----,(\mathrm{n}-2)$ and $\mathrm{j}=1,2,----,(\mathrm{m}-2)$

$\mu_{i}=1-\lambda_{i}=\frac{h_{i}}{h_{i}+h_{i+1}}$

$d_{i j}=\frac{6\left(\sigma_{i+1, j}-\sigma_{i, j}\right)}{h_{i}+h_{i+1}}$

Therefore equation (11) will become

$6\left[\sigma_{i+1, j}-\sigma_{i, j}\right\rfloor=2 M_{i+1}\left(h_{i}+h_{i+1}\right)+3 N_{j}\left(h_{i}+h_{i+1}\right)+M_{i} h_{i}+M_{i+2} h_{i+1}$

Dividing equation (14) by $h_{i}+h_{i+1}$ we get

$2 M_{i+1}+3 N_{j}+\mu_{i} M_{i}+\lambda_{i} M_{i+2}=d_{i j}$, wherei $=1,2,----,(\mathrm{n}-2)$ and $\mathrm{j}=1,2,-----,(\mathrm{m}-2)$

So the two variable natural cubic spline is 


$$
\begin{aligned}
S_{i j}= & \frac{M_{i}\left(x_{i+1}-x\right)^{3}}{6 h_{i}}+\frac{M_{i+1}\left(x-x_{i}\right)^{3}}{6 h_{i}}+\frac{N_{j}\left(y_{j+1}-y\right)\left(x-x_{i}\right)^{2}}{2 k_{j}}-\frac{N_{j+1}\left(y-y_{j}\right)\left(x_{i+1}-x\right)^{2}}{2 k_{j}} \\
& +\left\{\frac{\left(z_{i+1, j}-z_{i j}\right)}{h_{i} k_{j}}-\frac{\left(z_{i+1, j+1}-z_{i, j+1}\right)}{h_{i} k_{j}}+\frac{\left(N_{j+1}-N_{j}\right) h_{i}}{2 k_{j}}\right\}\left(y_{j+1}-y\right)\left(x-x_{i}\right)+ \\
& \left\{\frac{\left(M_{i+1}-M_{i}\right) h_{i}}{6}+\frac{N_{j+1} h_{i}}{2}-\frac{\left(z_{i+1, j+1}-z_{i, j+1}\right)}{h_{i}}\right\}\left(x_{i+1}-x\right)+ \\
& \left\{\frac{\left(z_{i, j+1}-z_{i j}\right)}{k_{j}}+\frac{N_{j+1} h_{i}^{2}}{2 k_{j}}\right\}\left(y-y_{j}\right)+\left\{\left(z_{i+1, j+1}-z_{i, j+1}\right)+z_{i j}-\left(\frac{M_{i+1}}{6}+\frac{N_{j+1}}{2}\right) h_{i}{ }^{2}\right\},
\end{aligned}
$$

$\forall(x, y) \in\left[x_{i}, x_{i+1}\right] \times\left\lfloor y_{j}, y_{j+1}\right\rfloor, \forall i=1,2, \ldots . .,(n-1), \forall j=1,2, \ldots \ldots .(m-1)$

where $2 M_{i+1}+3 N_{j}+\mu_{i} M_{i}+\lambda_{i} M_{i+2}=d_{i j}, \quad \mathrm{i}=1,2,----,(\mathrm{n}-2)$ and $\mathrm{j}=1,2,-----,(\mathrm{m}-2)$

For natural spline $M_{1}=M_{n}=N_{1}=N_{m}=0$

\section{Illustration}

Consider the two variable function $f(x, y)=e^{x y}$. The following table gives the function values for $\mathrm{x}$ taking values $0,0.1,0.2$ and y taking values $0,0.1,0.2$

\begin{tabular}{|l|l|l|l|}
\hline $\mathbf{X}$ & 0 & 0.1 & 0.2 \\
\hline 0 & 1 & 1 & 1 \\
\hline 0.1 & 1 & 1.01 & 1.02 \\
\hline 0.2 & 1 & 1.02 & 1.04 \\
\hline
\end{tabular}

$h_{1}=h_{2}=0.1$

$k_{1}=k_{2}=0.1$

Using (12) and (13),

$\sigma_{11}=0$

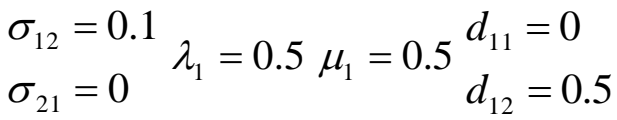

$\sigma_{22}=0.1$

Using (15) $\begin{aligned} & 2 M_{2}+3 N_{1}+\mu_{1} M_{1}+\lambda_{1} M_{3}=d_{11} \\ & 2 M_{2}+3 N_{2}+\mu_{1} M_{1}+\lambda_{1} M_{3}=d_{12}\end{aligned}$

For a natural cubic spline $\mathrm{M}_{1}=\mathrm{M}_{3}=\mathrm{N}_{1}=\mathrm{N}_{3}=0$

Solving (16) $\mathrm{M}_{2}=0$ and $\mathrm{N}_{2}=0.1667$

Using (8) the two variable cubic splines are

$$
S_{i j}(x, y)=\left\{\begin{array}{r}
-0.8335(y)(0.1-x)^{2}-0.9167(0.1-y)(x)-0.09167(0.1-x)+0.00834(y)+1.0092 \\
(x, y) \in I_{11} \\
0.8335(0.2-y)(x)^{2}-1.0834(0.2-y)(x)-0.2(0.1-x)+1.04,(x, y) \in I_{12} \\
-0.8335(y)(0.2-x)^{2}-0.9167(0.1-y)(x-0.1)-0.09167(0.2-x)+0.10834 y+1.0092 \\
(x, y) \in I_{21} \\
0.8335(0.2-y)(x-0.1)^{2}-1.0834(0.2-y)(x-0.1)-0.2(0.2-x)+0.1(y-0.1)+1.03 \\
(x, y) \in I_{22}
\end{array}\right.
$$

The following table shows the values of the function $\mathrm{f}$ at $(\mathrm{x}, \mathrm{y})$ and the corresponding interpolated values $S_{i j}(x, y)$ 


\begin{tabular}{|l|l|l|l|l|}
\hline & $(\mathbf{x}, \mathbf{y})$ & $S_{i j}(x, y)$ & $f(x, y)$ & Error \\
\hline 1 & $(0.05,0)$ & 1 & 1 & 0 \\
\hline 2 & $(0.05,0.05)$ & 1.003 & 1.003 & 0 \\
\hline 3 & $(0.15,0)$ & 1 & 1 & 0 \\
\hline 4 & $(0.15,0.15)$ & 1.022 & 1.022 & 0 \\
\hline 5 & $(0.05,0.15)$ & 1.007 & 1.007 & 0 \\
\hline
\end{tabular}

\section{Conclusion}

Interpolated values are found to be same as the actual functional value.

\section{References}

[1]. Anthony Ralston and Philip Rabinowitz, A first course in Numerical Analysis, Second Edition, Dover publication , INC, New York.

[2]. Ion Lixandru, Algorithm for the calculation of the two variables cubic spline function, AnaleleStiintifice Ale UniversitatiiMatematica, Tomul Lix,2013,f.1

[3]. Richard L Burden, J. Douglas Faires, Numerical Analysis, Ninth Edition,Brooks/Cole Cengage Learning.

[4]. S.S. Sastry, Introductory Methods Of Numerical Analysis, Fourth Edition, PHI Learning Private Limited 